\title{
Dimensionamento de vigas de concreto armadas à flexão com barras não metálicas
}

\begin{tabular}{|c|}
\hline GLÁUCIA MARIA DALFRÉ - ProfEsSORA \\
\hline FERNANDA GIANNOTTI DA SILVA FERREIRA - ProfESSORA \\
\hline AMANDA DUARTE ESCOBAL MAZZÚ - ENGENHEIRA CIVIL, DoutORANDA \\
\hline de Federal de São Carlos (PPGECI \\
\hline
\end{tabular}

\section{RESUMO}

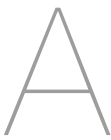

presenta-se $\mathrm{O}$ modelo do $\mathrm{ACl}$ 440.1R (2015) para o dimensionamento de elementos de concreto armados com barras de Polímeros Reforçados com Fibras (FRPs, Fiber Reinforced Polymers), considerando-se o fator ambiental de redução para diferentes materiais, as condições e premissas de dimensionamento, os procedimentos de cálculo e as verificações das três situações de ruína do elemento estrutural (esmagamento do concreto, ruptura da barra de FRP e ambas as situações).

\section{INTRODUÇÃO}

A substituição de armadura metálica pela armadura de FRP (Polímero Reforçado com Fibras - Fiber Reinforcement Polymer) altera o comportamento mecânico do elemento, sendo necessário que os procedimentos de dimensionamento sejam adaptados às propriedades da armadura não metálica. Como a utilização deste tipo de armadura ainda é recente, os procedimentos definidos ainda passarão por muitas discussões e mudanças. Sendo assim, é possível observar um conservadorismo na determinação dos limites de utilização deste material, além de divergências nos procedimentos desenvolvidos, uma vez que esses se baseiam sempre em normas de concreto armado convencional, que possuem procedimentos diferentes em cada local de aplicação.

Em estruturas armadas com barras de FRP, o dimensionamento é realizado como para as estruturas de concreto armadas com barras de aço, garantindo-se a segurança contra a ruína e a adequação às condições de utilização. Devido ao baixo módulo de elasticidade do material, as situações de serviço são as limitantes das estruturas de concreto armadas com barras de FRP, mas, ainda assim, o dimensionamento é realizado com base no Estado Limite Último (ELU), sendo, desta forma, semelhante ao método empregado para estruturas de concreto armadas com barras de aço.

Os procedimentos aqui apresentados para dimensionamento de estruturas armadas com FRP são norteados pela norma $\mathrm{ACl} 440.1 \mathrm{R}$ (2015), a qual é baseada no ACl 318 (2019).

\section{RECOMENDAÇÕES DE DIMENSIONAMENTO DAS BARRAS NÃO METÁLICAS À FLEXÃO SEGUNDO $O$ ACl 440.IR (2015)}

\section{I Estado limite último (ELU)}

Segundo Tavares (2006), com relação à durabilidade, as estruturas de concreto armadas com barras de FRP são tratadas de forma diferente das estruturas de concreto armadas com barras de aço, uma vez que não existe mais a preocupação com a corrosão da armadura, havendo apenas a necessidade de se garantir a durabilidade da armadura e a proteção da fibra utilizada, aspectos que refletem na vida útil da estrutura. Cada norma de dimensionamento, como o ACl 440.1R (2015), a norma norueguesa NS3473, o Canadian Highway Bridge Design Code (CHBDC), a Japan Society of Civil Engineers (JSCE), a norma holandesa BISE e a FIB 9.3 (Federation International du Béton), por exemplo, apresenta uma forma de tratamento para o fator ambiental de redução a ser utilizado no dimensionamento. 


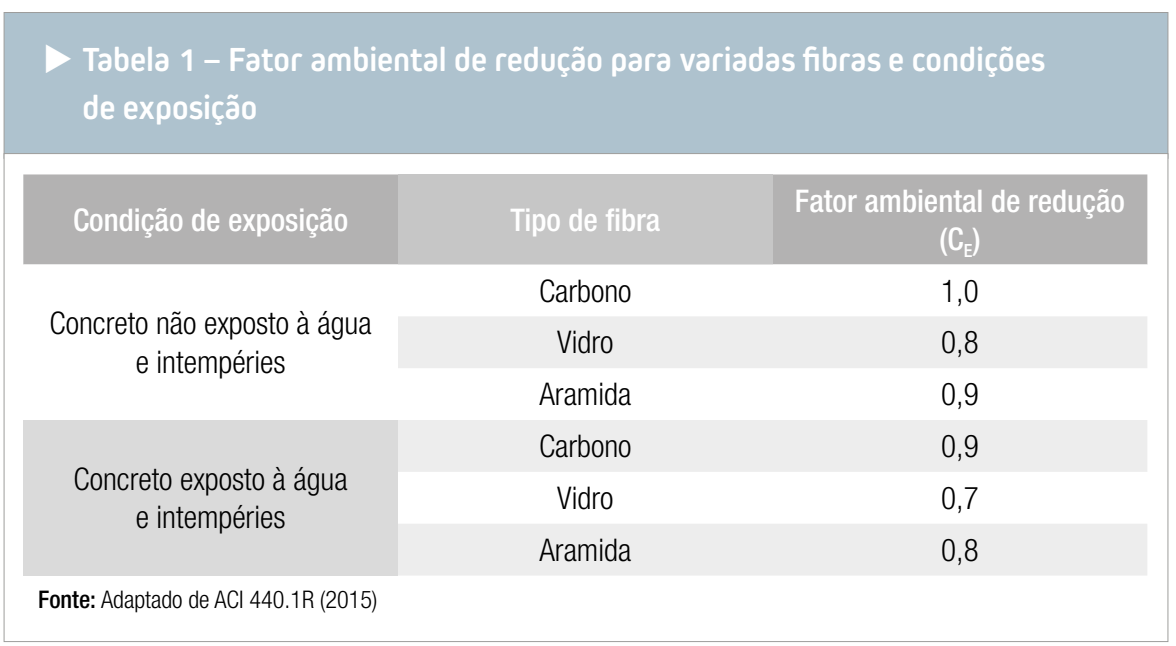

Na Tabela 1, correspondente à Tabela 6.2 do $\mathrm{ACl}$ 440.1R (2015), constam os valores do fator ambiental de redução das propriedades dos materiais estabelecidos por esta norma.

Para estruturas convencionais de concreto com armadura de aço o dimensionamento é realizado visando o escoamento da barra de aço, sendo esta considerada a melhor situação de dimensionamento. Desta forma, a capacidade resistente das barras é utilizada em sua totalidade, garantindo o escoamento e, consequentemente, a ductilidade da estrutura. Quando o elemento é dimensionado para a utilização de toda sua capacidade resistente, é chamado de subarmado. Se a tensão solicitante for muito menor que a capacidade da armadura, o elemento é chamado superarmado, sendo que a ruína da estrutura é dada pelo esmagamento do concreto comprimido.

Os FRPs não apresentam escoamento uma vez que seu comportamento é considerado elástico linear e frágil quando atingem toda a sua capacidade resistente, tal como o apresentado na Figura 1. Nesta Figura, CFRP corresponde aos Polímeros Reforçados com Fibras de Carbono (Carbon Fiber Reinforced Polymer), GFRP corresponde aos Polímeros Reforçados com
Fibras de Vidro (Glass Fiber Reinforced Polymer), AFRP aos Polímeros Reforçados com Fibras de Aramida (Aramid Fiber Reinforced Polymer) e BFRP aos Polímeros Reforçados com Fibras de Basalto (Basalt Fiber Reinforced Polymer), respectivamente.

Assim, para o dimensionamento de um elemento armado com barras de FRP não se pode levar em conta o escoamento das barras que compõem a armadura e a melhor situação de dimensionamento passa a ser a ruína do elemento por esmagamento do concreto na fibra mais comprimida. A forma mais econômica de dimensionamento é considerada quando o elemento atinge a ruína balanceada, na qual ocorrem, simultaneamente, a ruptura da armadura e o esmagamento do concreto. Neste caso, a linha neutra se localiza na parte superior da altura útil da seção transversal, próxima à superfície comprimida, como mostra a Figura 2 (TAVARES, 2006).

São dois os tipos de ruína que estruturas de concreto armadas com barras de FRP apresentam: ruptura da barra de FRP e esmagamento do concreto. No primeiro caso, a ruína é considerada repentina e brusca, devido ao fato de não existir escoamento das barras de FRP. A ruína por esmagamento do concreto é mais recomendável, pois ainda existe uma ductilidade dada pelo desenvolvimento do comportamento plástico do concreto antes da ruína (ACl 440.1R, 2015).

Algumas hipóteses devem ser consideradas para a simplificação do

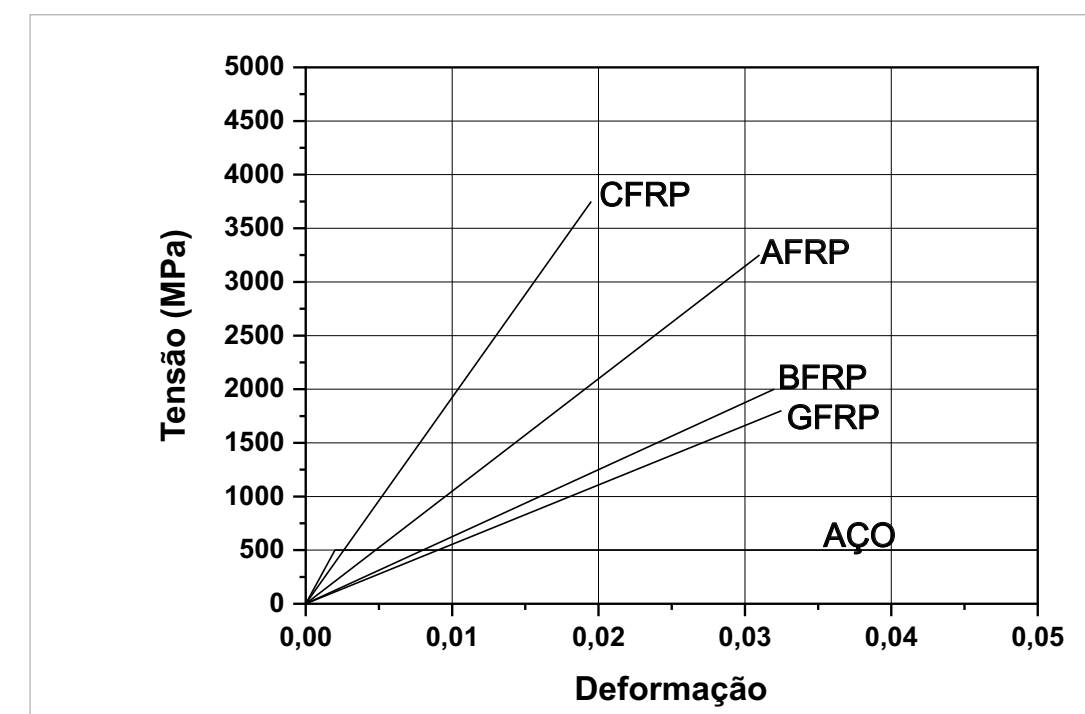

Fonte: Adaptado de FIB Bulletin 90 (2019)

Diagrama tensão versus deformação do aço e principais tipos de fibras 

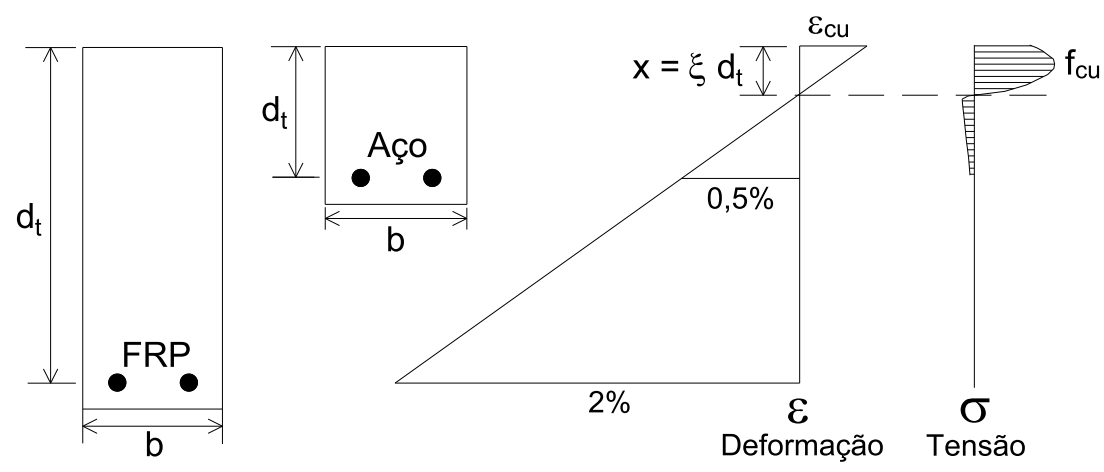

Fonte: Adaptado de Pilakoutas et al. (2002)

Figura 2

Distribuição das deformações para uma armadura de FRP

fenômeno real que ocorre nos elementos armados com barras de FRP:

Seções planas permanecem planas após a deformação do elemento;

- Deformação máxima do concreto antes da ruína é de $3 \%$;

- A capacidade resistente do concreto à tração não é considerada na resistência do elemento;

- O comportamento das barras de FRP é elástico linear até a ruptura; $e$

- Existe aderência perfeita entre o concreto e as barras de FRP.

Considerando-se a capacidade última de resistência, o dimensionamento de um elemento consiste em majorar as ações solicitantes (aquelas às quais o elemento está submetido) e minorar a capacidade resistente, garantindo-se, de forma segura, que a capacidade resistente não será menor do que as solicitações, tal como apresentado na Equação 1, onde $M_{n}$ é o momento resistente do elemento (calculado com o uso das equações de equilíbrio e a compatibilidade das deformações, dependente do tipo de ruína que governa o comportamento do elemento), $\mathrm{M}_{\mathrm{u}} \mathrm{O}$ momento calculado partindo-se das ações às quais o elemento está submetido, já multiplicadas pelos fatores de majoração probabilísticos definidos no ACl 318 (2019), e $\phi$, um coeficiente que depende do tipo de ruína que governa o comportamento da peça.

\begin{tabular}{|l|l|}
\hline$\phi \cdot M_{n} \geq M_{u}$ & 1 \\
\hline
\end{tabular}

Para a definição do tipo de ruína, parâmetros como a taxa de armadura de FRP do elemento e a taxa de armadura balanceada (taxa de armadura para a qual ocorrem, simultaneamente, ruptura do FRP e esmagamento do concreto) são utilizados. A taxa de armadura de FRP é dada pela Equação 2 e a taxa de armadura balanceada pela Equação 3. Como as barras de FRP não apresentam escoamento, a taxa de armadura balanceada é calculada usando a resistência à tração de projeto.

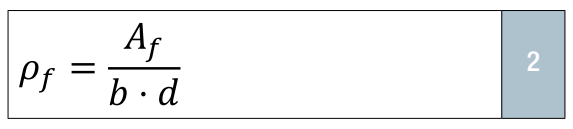

\begin{tabular}{|l|l|}
$\rho_{f b}=0,85 \cdot \beta_{1} \cdot \frac{f_{c}^{\prime}}{f_{f u}} \cdot \frac{E_{f} \cdot \varepsilon_{c u}}{E_{f} \cdot \varepsilon_{c u}+f_{f u}}$ & 3 \\
\hline
\end{tabular}

$\mathrm{Na}$ Equação 2, $A_{f}$ é a área das barras de FRP em $\mathrm{mm}^{2}$, b é a largura da viga de concreto em $\mathrm{mm}$ e d é a altura útil da viga de concreto, também em mm. Na Equação 3, $\beta_{1}$ é um fator de redução (com valor de 0,85 para concretos com resistência característica menor ou igual a 27,6 MPa e sofrendo reduções de 0,05 para cada 6,9 MPa extrapolado do limite de 27,6 MPa, nunca menor que 0,65), $f_{c}^{\prime}$ é a resistência característica à compressão do concreto, em $\mathrm{MPa}, \mathrm{E}_{\mathrm{f}}$ é o módulo de elasticidade de projeto da barra de FRP dado pelo fabricante, em MPa, $f_{f u}$ é a resistência à tração de projeto da barra de FRP, também em MPa, e $\varepsilon_{\text {cu }}$ é a deformação última no concreto em $\mathrm{mm} / \mathrm{mm}$.

Com relação à $\mathrm{f}_{\mathrm{fu}}$, tem-se a Equação 4, na qual $f_{f u}^{\star}$ é a resistência à tração da barra de FRP dada pelo fabricante ou o valor médio obtido experimentalmente em corpos de prova ensaiados $\left(f_{f u}^{*}=f_{f u, a v e}-3 \cdot \sigma\right.$, sendo $f_{f u, a v e}$ a resistência média à tração das amostras-teste, em MPa e $\sigma$ o desvio padrão, também em $\mathrm{MPa}$ ) e $\mathrm{C}_{\mathrm{E}}$ O fator ambiental de redução.

\begin{tabular}{|l|l|}
\hline$f_{f u}=C_{E} \cdot f_{f u}^{*}$ & 4 \\
\hline
\end{tabular}

Analogamente, tem-se as Equações 5 e 6, onde $\varepsilon_{\text {fu }}$ é a deformação de ruptura de projeto da barra de reforço de FRP, em \%, $C_{E}$ é o fator ambiental de redução e $\varepsilon_{f u}^{\star}$ é a deformação da barra de FRP fornecida pelo fabricante, em \%, ou calculada pela Equação 6, sendo $\varepsilon_{\text {u,ave }}$ a deformação média à tração das amostras-teste, em \%, e $\sigma$ o desvio padrão, também em \%.

$\varepsilon_{f u}=C_{E} \cdot \varepsilon_{f u}^{*}$

$\varepsilon_{f u}^{*}=\varepsilon_{u, a v e}-3 \cdot \sigma$

Conforme afirmam Feeser e Brown (2005), as propriedades do FRP variam mais entre os fabricantes do que as propriedades do aço. As barras de FRP não são padronizadas em toda a indústria de fabricação. A menos que o projetista exija um fabricante de FRP 
específico e um determinado tipo de especificação de barra, as barras de FRP utilizadas na construção terão propriedades diferentes das consideradas no projeto. Assim, os projetistas devem ter cuidado para especificar corretamente as propriedades necessárias das barras de FRP consideradas no projeto, de modo que sejam utilizadas barras de mesmas propriedades na execução.

O fator de redução da resistência, $\phi$, depende da razão entre a taxa de armadura de FRP $\left(\rho_{\mathrm{f}}\right)$ e a taxa de armadura balanceada $\left(\rho_{\mathrm{ff}}\right)$, como apresentado na Equação 7.

$\phi=\left\{\begin{array}{c|c|}0,55 \rightarrow \rho_{f} \leq \rho_{f b} & \\ 0,3+0,25 \cdot \frac{\rho_{f}}{\rho_{f b}} \rightarrow \rho_{f b}<\rho_{f}<1,4 \cdot \rho_{f b} & 7 \\ 0,65 \rightarrow \rho_{f} \geq 1,4 \cdot \rho_{f b} & \\ \hline\end{array}\right.$

A Figura 3 apresenta o gráfico do fator de redução da resistência em função da taxa de armadura de FRP. Se $\rho_{\mathrm{f}}<\rho_{\mathrm{fb}}$, a ruína é causada pela ruptura da barra. Se $\rho_{f}>1,4 \cdot \rho_{\mathrm{fb}}$, a ruína é causada pelo esmagamento do concreto. São três os casos possíveis para a ruína do elemento: ruína governada pelo esmagamento do concreto, ruína governada pela ruptura da barra e ruína governada pela ruptura da barra e esmagamento do concreto (zona de transição).

De acordo com Feeser e Brown (2005), em situações típicas de projeto em concreto armado, o projetista especifica uma resistência à compressão de concreto aos 28 dias. $\mathrm{Na}$ maior parte dos casos, o concreto utilizado excede a resistência especificada. Em elementos armados com FRP, uma resistência à compressão no concreto maior do que a especificada poderia (sob certas circunstâncias) resultar em uma mudança no modo de falha e, consequentemente,

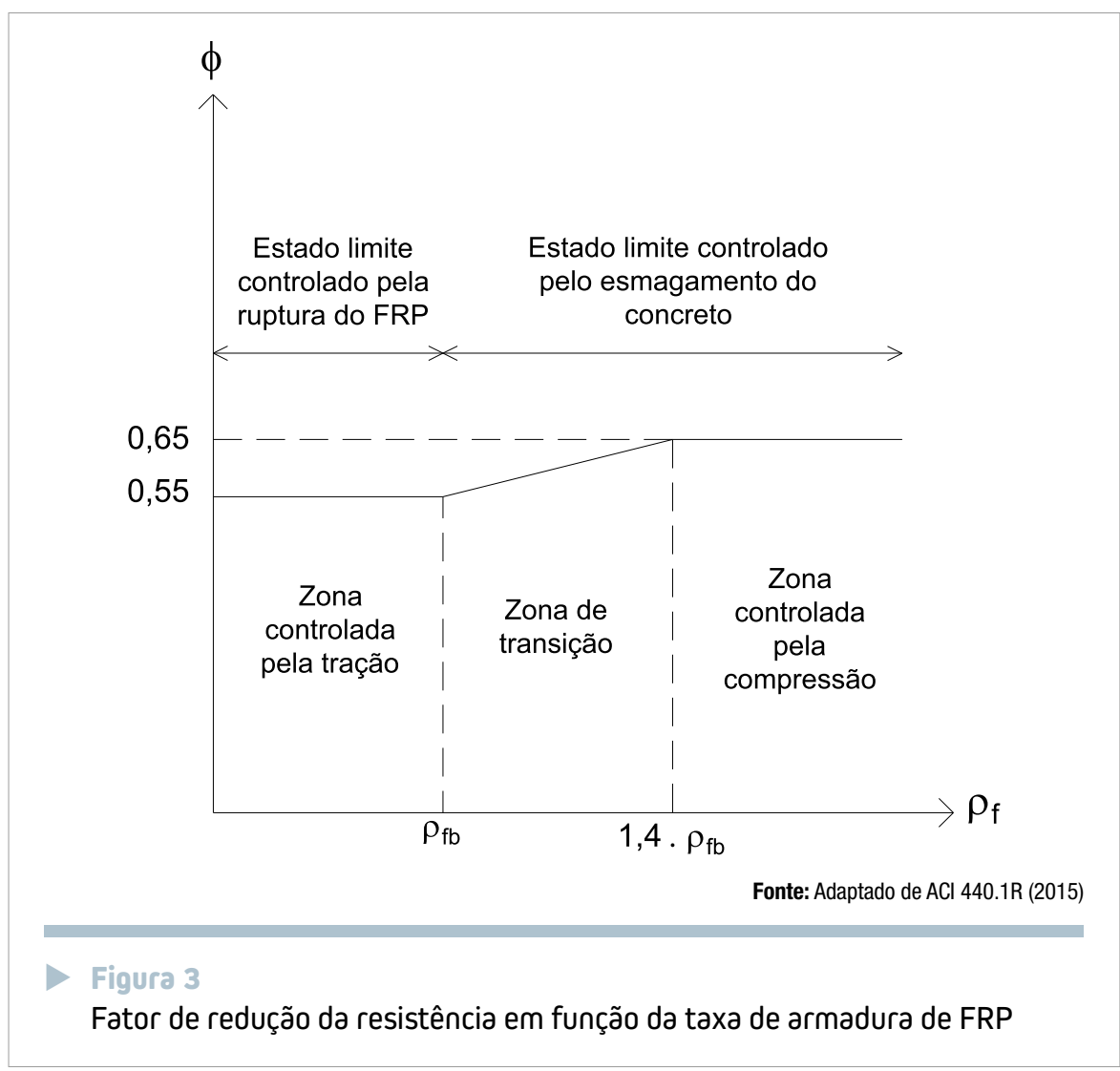

em uma mudança no fator de redução da resistência, alterando o valor de $\phi \cdot M_{n}$, utilizado para a comparação com as ações solicitantes.

\section{I.I RUINA GOVERNADA PELO ESMAGAMENTO DO CONCRETO}

Quando $\rho_{\mathrm{f}}>1,4 \cdot \rho_{\mathrm{fb}}$, O diagrama de tensões no concreto pode ser apro- ximado para a forma retangular apresentada no ACl 318 (2019), tal como apresentado na Figura 4.

Assim, por meio das equações de equilíbrio e da compatibilidade das deformações, é calculada a capacidade resistente do elemento. A força resultante na barra de FRP $\left(R_{f}\right)$ é descrita na Equação 8 (onde $A_{f}$ é a área de armadura de FRP e f é a tensão na
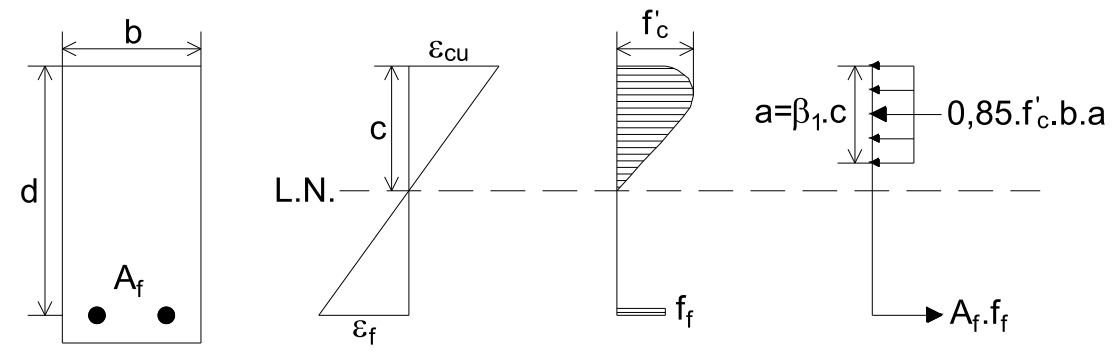

Fonte: Adaptado de ACl 440.1R (2015)

Figura 4

Diagrama aproximado retangular para esmagamento do concreto 
armadura de FRP) e o braço de alavanca (z) na Equação 9.

$$
\begin{array}{l|l|}
\hline R_{f}=A_{f} \cdot f_{f} & 8 \\
\hline z=d-\frac{a}{2} \text { ou } z=d-\frac{\beta_{1} \cdot c}{2} & 9 \\
\hline
\end{array}
$$

Multiplicando-se a força resultante na armadura e o braço de alavanca definido pelo binário de forças, obtém-se a resistência nominal à flexão, como mostram as Equações 10 a 18.

$$
\begin{array}{|l|l|}
\hline M_{n}=A_{f} \cdot f_{f} \cdot\left(d-\frac{a}{2}\right) & 10 \\
\hline
\end{array}
$$

Assim, do equilíbrio de forças, sendo $R_{c}$ a força resultante na região comprimida:

\begin{tabular}{|l|l|}
\hline$R_{f}=R_{c}$ & 11 \\
\hline
\end{tabular}

Portanto:

$A_{f} \cdot f_{f}=0,85 \cdot f_{c}^{\prime} \cdot b \cdot a$

Pela igualdade de forças, é definido o valor da altura do diagrama retangular equivalente:

$$
a=\frac{A_{f} \cdot f_{f}}{0,85 \cdot f_{c}^{\prime} \cdot b}
$$

Devido ao fato do diagrama tensão versus deformação das barras de FRP ser elástico linear, a Lei de Hooke pode ser aplicada. Assim, sendo $\varepsilon_{f}$ a deformação na barra de FRP, tem-se:

$$
f_{f}=E_{f} \cdot \varepsilon_{f}
$$

Utilizando-se a compatibilidade de deformações:

$$
\begin{array}{|l|l|}
\hline \frac{\varepsilon_{f}}{d-c}=\frac{\varepsilon_{c u}}{c} & 15 \\
\hline
\end{array}
$$

Isolando-se o valor da deformação na armadura:

$$
\varepsilon_{f}=\varepsilon_{c u} \cdot\left(\frac{d-c}{c}\right)
$$

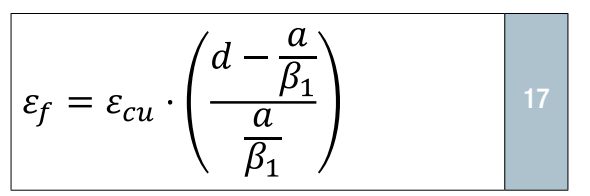

\begin{tabular}{|l|l|}
\hline$\varepsilon_{f}=\varepsilon_{c u} \cdot\left(\frac{\beta_{1} \cdot d-a}{a}\right)$ & 18 \\
\hline
\end{tabular}

Desta forma, fica determinado o valor da tensão na armadura de FRP, em função da deformação do FRP e do diagrama equivalente de tensões, o qual é apresentado na Equação 19.

\begin{tabular}{|l|l|}
\hline$f_{f}=E_{f} \cdot \varepsilon_{c u} \cdot\left(\frac{\beta_{1} \cdot d-a}{a}\right)$ & 19 \\
\hline
\end{tabular}

Fazendo-se a substituição da altura do diagrama equivalente (Equação 13) na Equação 19 e rearranjando-se os termos, tem-se a Equação 20:

$f_{f}=E_{f} \cdot \varepsilon_{c u} \cdot\left(\left(\left(\frac{b \cdot d}{A_{f}}\right) \cdot\left(\frac{\beta_{1} \cdot 0,85 \cdot f_{c}^{\prime}}{f_{f}}\right)\right)-1\right) 20$

Substituindo-se o valor da taxa de armadura (Equação 2) na Equação 20 e rearranjando-se os termos, obtém-se a equação do $2^{\circ}$ grau expressa na Equação 21.

\begin{tabular}{|l|l|}
\hline$\rho_{f} \cdot f_{f}^{2}+E_{f} \cdot \varepsilon_{c u} \cdot \rho_{f} \cdot f_{f}$ \\
$-E_{f} \cdot \varepsilon_{c u} \cdot \beta_{1} \cdot 0,85 \cdot f_{c}^{\prime}=0$
\end{tabular}

Resolvendo-se a Equação $21 \mathrm{em}$ termos da tensão na armadura de FRP e considerando-se apenas a raiz positiva da equação, obtém-se a Equação 22.

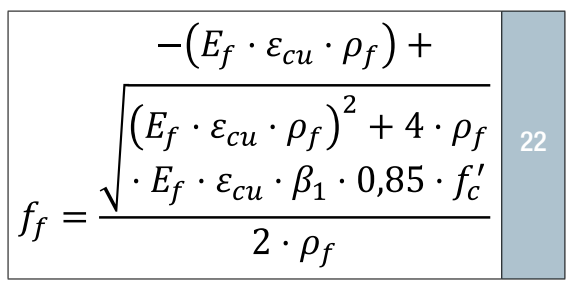

Rearranjando-se os termos da Equação 22 é possível obter a Equação 23 para o cálculo da tensão na armadura de FRP, a qual não deve nunca ser superior ao valor da resistência à tração de projeto da barra de FRP.

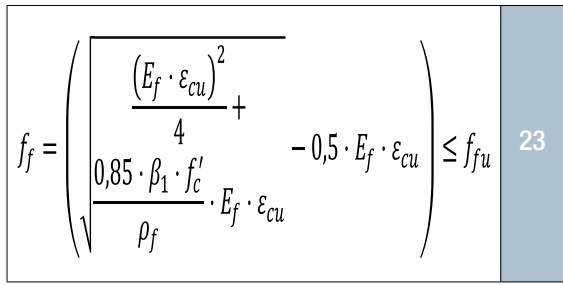

Por fim, substituindo-se a altura do diagrama equivalente (Equação 13) na Equação 10, obtém-se a Equação 24 para o cálculo do momento resistente nominal, em termos da área de armadura de FRP, a qual resulta na Equação 25 após o rearranjo dos termos.

$M_{n}=A_{f} \cdot f_{f} \cdot\left(d-\left.\left(\begin{array}{c}\left(\frac{1}{2 \cdot 0,85}\right) \\ \left.\cdot\left(\frac{A_{f} \cdot f_{f}}{f_{c}^{\prime} \cdot b}\right)\right)\end{array}\right)\right|^{24}\right.$

$M_{n}=A_{f} \cdot f_{f}$.

$\left(d-0,59 \cdot\left(\frac{A_{f} \cdot f_{f}}{f_{c}^{\prime} \cdot b}\right)\right)$

Isolando-se a área de armadura de FRP na Equação 2 e substituindo-se na Equação 25, obtém-se a Equação 26 para o cálculo do momento resistente nominal em termos da taxa de armadura de FRP.

$$
\begin{aligned}
& M_{n}=\rho_{f} \cdot f_{f} \cdot \\
& \left(1-0,59 \cdot \frac{\rho_{f} \cdot f_{f}}{f_{c}^{\prime}}\right) \cdot b \cdot d^{2}
\end{aligned}
$$

\section{I.2 RUÍNA gOVERNADA PELA RUPTURA DA BARRA DE FRP}

Quando $\rho_{\mathrm{f}}<\rho_{\mathrm{fb}}$, o diagrama de tensões no concreto não pode ser aproximado para a forma retangular apresentada no ACl 318 (2019), uma vez que a deformação máxima do concreto não pode ser atingida $\left(\varepsilon_{\mathrm{c}}<\varepsilon_{\mathrm{cu}}\right)$. Assim, um diagrama retangular equivalente deve 
ser calculado, como mostra a Figura 5. Este diagrama apresenta como incógnitas a deformação final no concreto quando o FRP rompe e a distância da fibra mais comprimida à linha neutra. Os fatores $\alpha_{1}$ e $\beta_{1}$ (Equações 27 e 28), que definem o diagrama retangular equivalente, também são desconhecidos.

\begin{tabular}{|l|l|}
\hline$\alpha_{1}=\frac{f_{c m}}{f_{c}^{\prime}}$ & 27 \\
\hline
\end{tabular}

\begin{tabular}{|l|l|}
\hline$\beta_{1}=\frac{a}{c}$ & 28 \\
\hline
\end{tabular}

Para efeito de simplificação, uma vez que a análise do diagrama envolvendo todas as variáveis é muito complexa, a resistência nominal à flexão pode ser obtida pela Equação 29.

$M_{n}=A_{f} \cdot f_{f u} \cdot\left(d-\frac{\beta_{1} \cdot c}{2}\right)$

Para uma dada seção, o produto $\beta_{1}$. c varia, dependendo das propriedades do material e da relação da taxa de armadura de FRP. Para uma seção controlada pelo Estado Limite de Ruptura de FRP, o valor máximo para este produto é $\beta_{1} \cdot c_{b}$ e é atingido quando a deformação máxima de 0,003 no concreto é atingida, tal como apresentado nas Equações 30 e 31.

$$
\begin{array}{|l|l|}
\hline M_{n}=A_{f} \cdot f_{f u} \cdot\left(d-\frac{\beta_{1} \cdot c_{b}}{2}\right) & 30 \\
\hline
\end{array}
$$

\begin{tabular}{|l|l|}
\hline$c_{b}=\left(\frac{\varepsilon_{c u}}{\varepsilon_{c u}+\varepsilon_{f u}}\right) \cdot d$ & 31 \\
\hline
\end{tabular}

\section{I.3 Ruína governada PELA RUPTURA DA BARRA $\in$ ESMAGAMENTO DO CONCRETO}

Este caso é uma forma mais conservadora de se considerar a ruína governada pela ruptura da barra de FRP, no qual
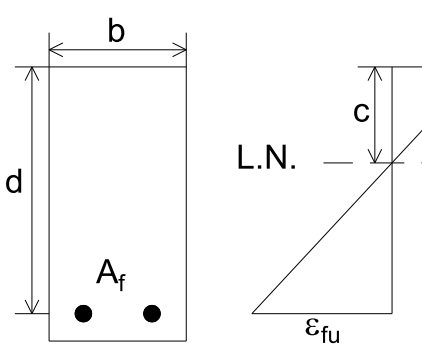

$\varepsilon_{\mathrm{C}}$
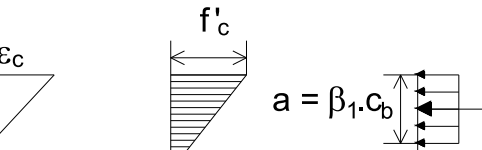

$0,85 . f_{c} ' \cdot b . a$

Figura 5

Diagrama equivalente para ruptura da barra de FRP a linha neutra é levada para um ponto onde ocorra a ruína governada pelos dois fatores em conjunto. Se $\rho_{\mathrm{f}}<\rho_{\mathrm{fb}}<1,4 \cdot \rho_{\mathrm{fb}}$, o dimensionamento é dito balanceado e a ruína da estrutura acontece pela ruptura da barra e esmagamento do concreto simultaneamente (Figura 6).

Assim, sabendo-se que $c=a / \beta_{1}$, têm-se as Equações 32 e 33.

$$
\begin{array}{|l|l|}
\hline \frac{\varepsilon_{f u}}{d-c}=\frac{\varepsilon_{c u}}{c} & 32 \\
\hline
\end{array}
$$

$c=\left(\frac{\varepsilon_{c u}}{\varepsilon_{c u}+\varepsilon_{f u}}\right) \cdot d$

O momento resistente será o menor

$M_{n} \leq\left\{\begin{array}{c}A_{f} \cdot f_{f u} \cdot\left(d-\frac{a}{2}\right) \\ 0,85 \cdot f_{c}^{\prime} \cdot b \cdot \beta_{1} \cdot c \cdot\left(d-\frac{a}{2}\right)\end{array}{ }^{34}\right.$

\section{I.4 ARMadura míNIma}

O limite mínimo de armadura é cal- culado apenas para a situação em que a ruína do elemento é governada pela ruptura da barra de FRP $\left(\rho_{\mathrm{f}}<\rho_{\mathrm{ff}}\right)$, e é utilizado para evitar a ruptura por fissuração do concreto, tal como apresentado na Equação 35, na qual $\mathrm{M}_{\mathrm{cr}}$ é o momento de fissuração do concreto.

$\phi \cdot M_{n} \geq M_{c r}$

As recomendações do ACl 318 (2019) para o limite mínimo de armadura são baseadas neste mesmo conceito e podem ser aplicadas, com modificações, para o caso da utilização de armadura de FRP. Tais modificações são baseadas na diferença entre os coeficientes de redução da resistência $(\phi)$ dados pelo ACl 318 (2019) e pelo $\mathrm{ACl} 440.1 \mathrm{R}$ (2015), sendo igual a 0,9 e 0,55 para tais normas, respectivamente. Com $\phi=0,55$ e a multiplicação da equação do ACl 318 (2019) existente para a armadura de aço (Equação 36) por 1,64 (0,9/0,55 = 1,64), tem-se o apresentado na Equação 37. Na Equação 36,

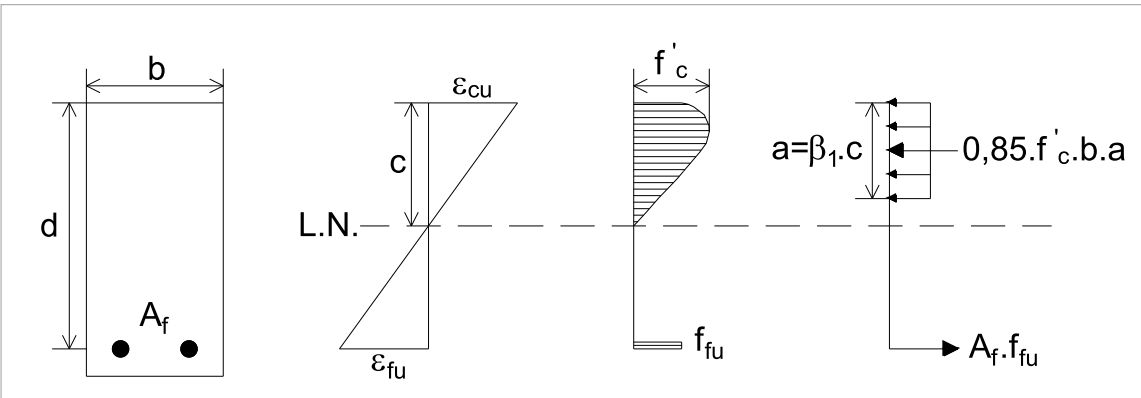

Fonte: Adaptado de ACl 440.1R (2015)

Diagrama equivalente para ruptura balanceada 
$\mathrm{f}_{\mathrm{y}}$ corresponde a tensão de escoamento do aço, enquanto na Equação 37, é utilizado o valor da resistência à tração de projeto da barra de FRP. Destaca-se que na Equação 37 se utiliza f' ${ }_{\text {c }}$ em MPa, $\mathrm{f}_{\mathrm{fu}}$ em MPa, b em mm e d em mm, obtendo-se $A_{f, \text { min }}$ em mm².

\begin{tabular}{|l|l|}
$A_{s, \text { min }}=\frac{0,25 \cdot \sqrt{f_{c}^{\prime}}}{f_{y}}$. \\
$b \cdot d \geq \frac{1,4}{f_{y}} \cdot b \cdot d$
\end{tabular}

\begin{tabular}{|l|l|}
$A_{f, \text { min }}=\frac{0,41 \cdot \sqrt{f_{c}^{\prime}}}{f_{f u}}$. & $37^{\prime}$ \\
$b \cdot d \geq \frac{2,3}{f_{f u}} \cdot b \cdot d$ & \\
\hline
\end{tabular}

\subsection{Estado Limite de Serviço (ELS)}

Elementos de concreto armados com barras de FRP apresentam uma rigidez, após a fissuração, menor do que elementos armados com barras de aço. Consequentemente, deformações pela ação das cargas de serviço são maiores e podem acabar controlando o projeto. De maneira geral, os elementos armados com barras de FRP podem acabar não satisfazendo os critérios estabelecidos para a deformação e a abertura de fissuras.

A capacidade de serviço pode ser definida como o desempenho satisfatório em condições de cargas de serviço e pode ser descrita em termos de dois critérios:

Fissuração: A largura excessiva das fissuras é indesejável por razões estéticas e outras que podem danificar ou deteriorar o concreto estrutural;

> Deformação: As flechas devem estar dentro de limites aceitáveis impostos pela utilização da estrutura.

De acordo com Tavares (2006), uma vez que existe diferença de rigidez entre elementos armados com barras de aço e elementos armados com barras de FRP, a verificação dos limites de serviço não pode ser a mesma para esses elementos. Após o carregamento, um elemento armado com barras de FRP apresentará maior deformação e maior abertura de fissuras devido à sua menor rigidez. A formulação para verificação de ELS pode ser encontrada em Mazzú (2020).

\section{CONCLUSÕES}

Recentemente, assuntos relacionados à durabilidade, manutenção e reforço de estruturas de concreto armado têm se destacado em meio à indústria da construção civil. Neste cenário, materiais alternativos conhecidos como compósitos FRP (Fiber Reinforced Polymer, em língua inglesa) se destacam por suas excelentes propriedades mecânicas, como resistência, rigidez $e$ baixo peso próprio. Por esse motivo, este trabalho fez uma breve apresentação das barras não metálicas para uso como armadura de flexão em elementos de concreto armado.

Para além disso, devido à inexistência de normas brasileiras, apresentou-se o modelo de dimensionamento do $\mathrm{ACl}$ 440.1R (2015) para o dimensionamento com barras de FRP, os coeficientes de segurança e todo o procedimento de cálculo e verificações a ser realizadas.

${ }^{1}$ A OBTENÇÃO DE $\mathrm{f}_{\mathrm{fu}}$ FOI APRESENTADA NA EQUAÇÃO 4 E DEPENDE DA AGRESSIVIDADE AMBIENTAL E DA TENSÃO DE TRAÇÃO DADA PELO FABRICANTE OU OBTIDA EXPERIMENTALMENTE. TAL VALOR GERALMENTE É DADO EM MPA. COM RELAÇĀO AS UNIDADES PARA VERIFICAÇÃO DE $A_{s, \text { min }}$, NA PRIMEIRA VERIFICAÇÃO $\left(0,41 \cdot \sqrt{f_{c}^{\prime}} \cdot b \cdot d / f_{f u}\right)$ A ÁREA É DADA EM Mm ${ }^{2}$. NA SEGUNDA $\left(2,3 \cdot b \cdot d / f_{f u}\right)$ EXISTE UM FATOR

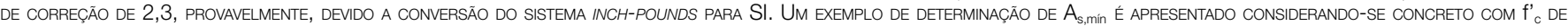
28 MPA, BARRA COM f $f_{f u}$ DE 496 MPa, b DE 250 MM E d IGUAL A 341MM.

$$
\begin{gathered}
A_{f, \text { min }}=\frac{0,41 \cdot \sqrt{f_{c}^{\prime}}}{f_{f u}} \cdot b \cdot d \geq \frac{2,3}{f_{f u}} \cdot b \cdot d \quad A_{f, \text { min }}=\frac{0,41 \cdot \sqrt{28 M P a}}{496 M P a} \cdot 250 \mathrm{~mm} \cdot 341 \mathrm{~mm} \geq \frac{2,3}{496 M P a} \cdot 250 \mathrm{~mm} \cdot 341 \mathrm{~mm} \\
A_{f, \text { mín }}=372,89 \mathrm{~mm}^{2} \geq 395,31 \mathrm{~mm}^{2} \quad A_{f, \text { mín }}=395,31 \mathrm{~mm}^{2}
\end{gathered}
$$

\section{> REFERÊNCIAS BIBLIOGRÁFICAS}

[01] AMERICAN CONCRETE INSTITUTE. ACI COMMITTEE 318. Building code requirements for reinforced concrete. Detroit, USA. 2019.

[02]__ ACI COMMITTEE 440.1R. Guide for the Design and Construction of Structural Concrete Reinforced with Fiber-Reinforced Polymer (FRP) Bars. 2015.

[03] FÉDÉRATION INTERNATIONALE DU BÉTON. FIB BULLETIN 90. Externally applied FRP reinforcement for concrete structures - Technical report. Alemanha, 2019.

[04] FEESER, W. K.; BROWN, V. L. Guide Examples for Design of Concrete Reinforced with FRP Bars. In: INTERNACIONAL SYMPOSIUM OF FIBER-REINFORCED POLYMER REINFORCEMENT FOR CONCRETE STRUCTURES, 7., v. 230, p. 935-954, 2005, Kansas. Anais... Kansas, 2005.

[05] MAZZÚ, A. D. E. Estudo sobre a substituição de armadura metálica por barras de GFRP em vigas de concreto armadas à flexão. Dissertação. Programa de Pós-Graduação em Engenharia Civil. Universidade Federal de São Carlos. São Carlos, 2020.

[06] PILAKOUTAS, K.; NEOCLEOUS, K.; GUADAGNINI, M. Design Philosophy Issues of Fiber Reinforced Polymer Reinforced Concrete Structures. Journal of Composites for Construction, v. 6, n. 3, Agosto, 2002.

[07] TAVARES, D. H. Análise teórica e experimental de vigas de concreto armadas com barras não metálicas de GFRP. São Carlos, 128p. Dissertação de Mestrado, Universidade de São Paulo, 2006 